\title{
X-Ray Imaging Calibration for Fuel-Coolant Interaction Experimental Facilities
}

\author{
Christophe Journeau ${ }^{1}$, Michael Johnson ${ }^{1,2}$, Shifali Singh ${ }^{1,3}$, Fréderic Payot ${ }^{1}$, \\ Ken-ichi Matsuba ${ }^{4}$, Yuki Emura ${ }^{4}$ and Kenji Kamiyama ${ }^{4}$ \\ ${ }^{1}$ CEA, DES, IRESNE, Cadarache, 13108 St-Paul-lez-Durance, France \\ ${ }^{2}$ SCALIAN DS, 399 Avenue du Château de Jouques, 13420 Gémenos, France \\ ${ }^{3}$ STROBE, INSERM (UA 7), Université Grenoble Alpes, Grenoble, France \\ ${ }^{4}$ JAEA, Oarai Research and Development Center, 4002 Narita, Oarai, Ibaraki 311-1393, Japan \\ christophe.journeau@cea.fr
}

\begin{abstract}
During a severe accident in either sodium-cooled or water-cooled nuclear reactors, jets of molten nuclear fuel may impinge on the coolant resulting in fuel-coolant interactions (FCI). Experimental programs are being conducted to study this phenomenology and to support the development of severe accident models. Due to the optical opacity of the test section walls, sodium coolant, and the apparent optical opacity of water in the presence of intense ebullition, high-speed X-ray imaging is the preferred technique for FCI visualization. The configuration of these X-ray imaging systems, whereby the test section is installed between a fan-beam X-ray source and a scintillator-image intensifier projecting an image in the visual spectrum onto a high-speed camera, entails certain imaging artefacts and uncertainties. The $\mathrm{X}$-ray imaging configuration requires precise calibration to enable detailed quantitative characterization of the FCI. To this end, 'phantom' models have been fabricated using polyethylene, either steel or hafnia powder, and empty cavities to represent sodium, molten fuel and sodium vapor phases respectively. A checkerboard configuration of the phantom enables calibration and correction for lens distortion artefacts which magnify features towards the edge of the field of view. Polydisperse steel ball configurations enable precise determination of the lower limit of detection and the estimation of parallax errors which introduce uncertainty in an object's silhouette dimensions. Calibration experiments at the MELT facility determined lower limits of detection in the order of $4 \mathrm{~mm}$ for steel spheres, and 1.7-3.75 mm for vapor films around a molten jet.
\end{abstract}

Keywords -X-Ray imaging, calibration, phantom, jet fragmentation, fuel-coolant interaction.

\section{INTRODUCTION}

$\mathrm{D}$ URING a postulated severe accident in either a Sodiumcooled Fast Reactor or a Light Water Reactor, molten core material (corium) may be discharged to the lower plenum and interact with the coolant. The progression of the subsequent fuel-coolant interaction (FCI) is likely to differ between corium-water [1-3] and corium-sodium interaction [4-6], however in each case the jet fragmentation will dictate the conditions for fuel-coolant contact and the mode of heat transfer, and may lead in some conditions to vapor explosions.

Due to the optical opacity of sodium, and of water in the presence of a large flux of bubbles, high-speed X-ray imaging has been the preferred approach for the visualization of FCIs [7-9]. CEA has developed SPECTRA (Software for Phase Extraction and Corium TRacking Analysis) [6] for the analysis of X-ray images acquired during FCI experiments, including the suppression of imaging artefacts and the quantitative characterization of the melt fragment population, in support of the development of the corium-sodium interaction code SCONE [10] .

The use of fan-beam X-ray imaging entails certain imaging artefacts, not limited to vignetting, due to the radial decay in photon intensity away from the axis of the fan-beam [9] [11] , lens distortion and additive white noise. In order to quantitatively interpret the progression of an FCI from X-ray image processing, calibration of the X-ray imaging system is recommended. The calibration of high-speed X-ray imaging systems using various 'phantom' models is presented herein. These phantoms provide images containing features of known geometries and with attenuation properties representative of the phases observed during the FCI. This calibration procedure enables a geometric calibration of the imaging system, achieving a homogeneous pixel resolution across the image, as well as the estimation of the observed X-ray energy within the test section and the lower limit of detection for small particles presenting with intensities close to the threshold contrast to noise ratio. Calibration results are presented for the Japanese MELT facility and the French KROTOS facility.

\section{GEOMETRIC CALIBRATION}

\section{A. Phantom design}

The choice of analogue materials for the phantoms is largely dictated their linear X-Ray attenuation coefficients and their suitability for the fabrication of complex geometries. At high $\mathrm{X}$-ray energies, the energy dependence of mass attenuation coefficients diminishes and the disparity in mass attenuation coefficient between potential analogues is reduced, and so finding analogue materials with appropriate linear attenuation coefficient becomes increasingly dependent on the material density [12]. Inert analogue materials were chosen with linear attenuation coefficients as close as possible to those of the 
relevant phases observed during FCI experiments:

- Thermoplastics, such as polyoxymethylene (POM) and polyethylene (PE), have attenuation behavior comparable to liquid sodium at photon energies $>100 \mathrm{keV}$, while offering sufficient versatility for the construction of complex phantom geometries. PE also represents a good simulant of liquid water in this energy spectrum.

- The melt phase can be represented by stainless steel 316 for metallic fuels or hafnia for oxidic fuels, due to its comparable density to $\mathrm{UO}_{2}$.

- Coolant vapor is represented by air.

At the $9 \mathrm{MeV}$ X-ray source energy employed at the KROTOS facility, the mass attenuation coefficient of a 50:50 uranium dioxide-stainless steel melt mixture is $0.038 \mathrm{~cm}^{2} \mathrm{~g}^{-1}$ while that of stainless steel is $0.030 \mathrm{~cm}^{2} \mathrm{~g}^{-1}$. The mass attenuation coefficient of sodium will be $0.0232 \mathrm{~cm}^{2} \mathrm{~g}^{-1}$ compared to $0.0238 \mathrm{~cm}^{2} \mathrm{~g}^{-1}$ for polyethylene [12] .

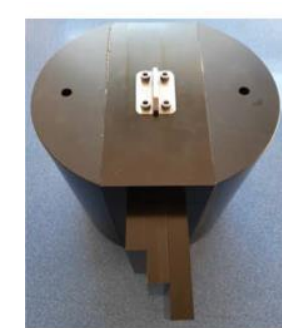

(a)

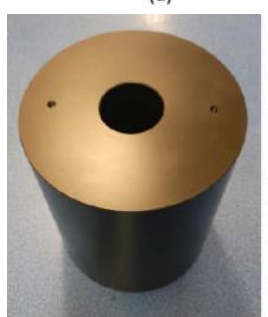

(h)

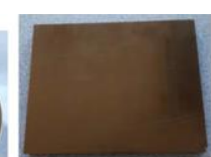

(b)

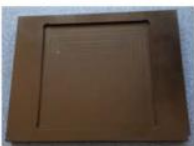

(e)

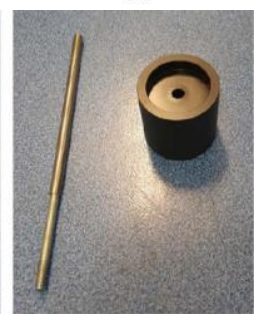

(i)

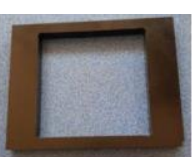

(c)

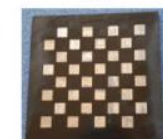

(f)

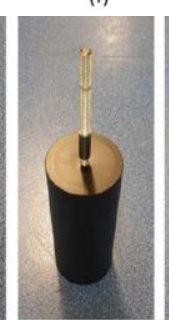

(j)

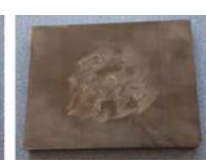

(d)

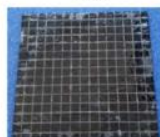

(g)

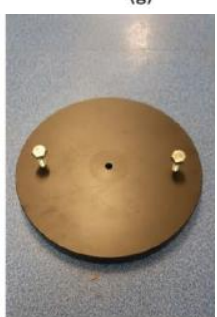

(k)
Fig. 1. Components of phantom 1 (a-g) and phantom 2 (h-k) used for calibration of the X-Ray imaging system at the MELT facility.

Fig. 1 presents the components of two phantoms which have been used for the calibration of the X-ray imaging system employed at the MELT facility at the JAEA's Oarai Center [6] . Both phantoms have $250 \mathrm{~mm}$ diameter, $280 \mathrm{~mm}$ length cylindrical geometries. Phantom 1 (Fig. 1a-g) has been designed such that interchangeable inserts can be installed within a void at the center of the cylinder, each designed with the purpose of calibrating a unique aspect of the imaging system. For example, the checkerboard inserts in Fig. 1j-g are designed to calibrate lens distortion artefacts, while an insert embedded with polydisperse steel balls (Fig. 1d) is used to determine the lower limit of detection, or the smallest detectable spherical melt objects. The interchangeability of the inserts allows objects of interest to be moved towards, or away from the X-ray source for the calibration of parallax uncertainties. Phantom 2 (Fig. 1h-k), has a smaller cylindrical void at its center for the insertion of a smaller phantom consisting of four concentric annular rings. Each of these annular rings can be selectively filled with fuel analogue to mimic different stages of jet pre-mixing.

\section{B. Noise and vignetting artefacts}

Dark current images are acquired without X-ray illumination and provide information on pattern and shot noise associated with the camera electronics. The time-averaged and denoised dark current images reveal the pattern noise which is used to offset the X-ray images prior to further image processing. Local denoising filters (mean, median or Gaussian) are generally avoided as smaller objects are likely to be obscured, phase boundaries blurred and the fine textures of complex melt fragment geometries are likely to be lost. Video-block matching and 3D filtering (V-BM3D) [13] and non-local means, NLM [14] [15] filters are preferred due to their selectivity in distinguishing between noise and real textures, rather than averaging local variations indiscriminately.

Flat field images represent X-ray images of the test section filled with coolant at rest, or a solid cylinder of thermoplastic coolant analogue for the purpose of the calibration experiments. Vignetting artefact represents the observed decay of pixel intensity away from the image center towards the border in the radiography of a homogeneous object, and is caused by the decay in photon flux away from the axis of the fan-beam X-ray source. Homogeneous illumination of the image is essential to achieve accurate segmentation of melt fragments and vapor clouds, independent of their location. Normalization of the dark current offset X-ray images by the flat field corrects for the heterogeneity in the illumination of the field of view [16] :

$$
I_{F F N}=k_{e q} \ln \left|\frac{I-I_{D C}}{I_{F F}-I_{D C}}\right|
$$

where $I_{F F N}$ is the log-flat field normalized image, $I$ is the original projected $\mathrm{X}$-ray image, $I_{D C}$ is the pattern noise of the dark current, $I_{F F}$ is the flat field image and $k_{e q}$ is an equalization factor employed to broaden the intensity histogram so that the image can be cast as an integer class (usually 8-bit or 16-bit) in order to reduce the computational expense of subsequent image processing.

Fig. 2a presents a schematic of phantom 2 installed within the test section and Fig. $2 b$ is a corresponding X-ray image (denoised and temporally-averaged for ease of visualization) of the phantom with its two inner annuli filled with stainless steel balls, while the two outer rings are void, thus representing a cloud of fragmented melt particles surrounded by vapor. Fig. $2 b$ demonstrates the decay in intensity towards the periphery of the circular field of view illuminated by the X-ray source. This is better demonstrated by Fig. 3a-b, which present the intensity signal over the horizontal analysis line shown in green Fig. 2b, before and after denoising respectively. The intensity signal of the coolant decays from around 60 near the center of the image to zero at the right-hand border and 20 at the left-hand border, demonstrating the asymmetry of the vignetting artefact. 

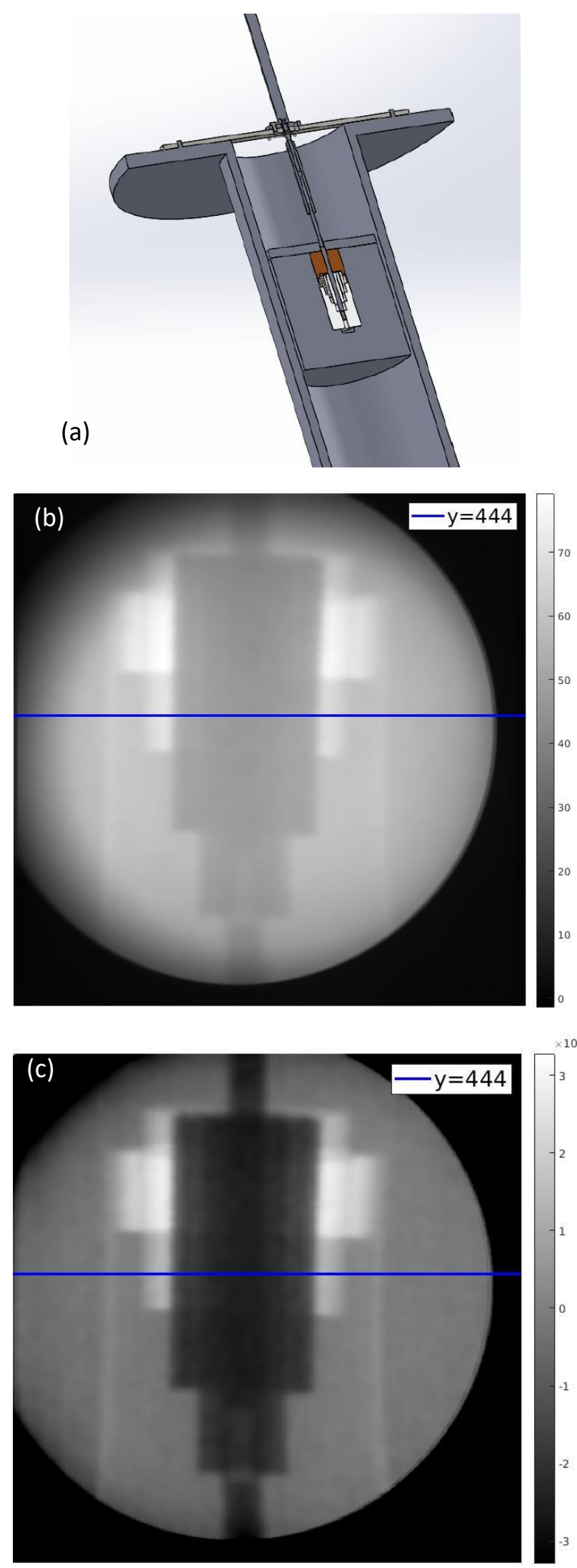

Fig. 2. (a) A schematic of phantom 2 installed in the test section, (b) a denoised X-ray image of phantom 2 with its 2 inner rings filled with steel balls, and (c) a $\log$-flat field normalized version of (b) equalized by a factor of $10^{5}$.
The resulting image, following log-flat field normalization, is presented in Fig. 2b, with the corresponding intensity signal shown in Fig. 3c. The plastic coolant regions of the log-flat field normalized image present with an intensity close to zero. The mild residual vignetting in Fig. $3 \mathrm{c}$ is due to subtle misalignment of the phantom between the calibration image and the flat field image, which would not be observed in the FCI experimental images. The void regions either side of the melt analogue exhibit elevated intensities and the steel-filled regions present with negative intensities. The three phases are much more easily distinguished in Fig. 2c and Fig. 3c following denoising and log-flat field normalization.

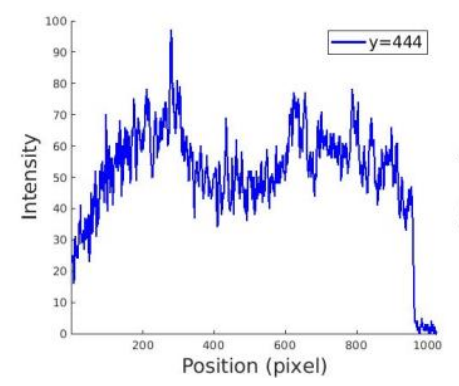

(a)

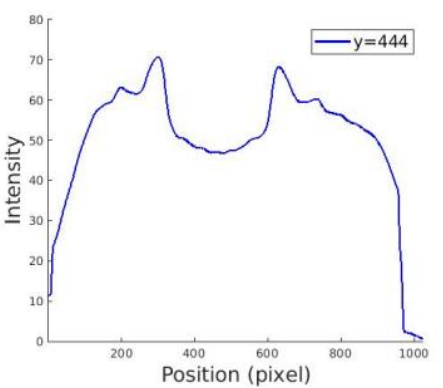

(b)

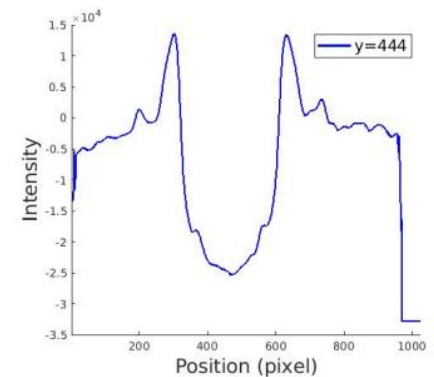

(c)

Fig. 3. Intensity profiles across the width of the phantom 2 images presented in Fig. 2b-c. The intensity signals correspond to images of the phantom (a) prior to denoising, (b) after denoising, and (c) after denoising and log-flat field normalization.

\section{Distortion artefacts}

In order to correct for distortion artefacts, X-ray images are acquired of checkerboard inserts installed in phantom 1. The coarse checkerboard (Fig. 1f) is constructed of $14.5 \times 14.5 \times 4 \mathrm{~mm}$ plastic and steel cuboids. The fine-wire checkerboard (Fig. 1g) is made of 14 horizontal and 14 vertical $1 \mathrm{~mm}$ diameter steel wires embedded in POM at regular $10 \mathrm{~mm}$ intervals. X-ray images of the two checkerboards are presented in Fig. 4. The fine-wire checkerboard in Fig. 4b, while fainter in contrast between the steel and plastic, was more effective in detailing the geometric distortion of the X-ray images. From the observed curvature of the vertical and horizontal wires in Fig. $4 \mathrm{~b}$, the origin of radial distortion was identified. The radial component of the lens distortion can be corrected using the approach detailed by Vass and Perlaki [17]. If $r$ and $\hat{r}$ are the distorted and undistorted radii from the center of distortion, the translation function to correct for radial distortion is found from a truncated Taylor-series expansion:

$$
\hat{r}=r\left(1+k r^{2}\right) \text {. }
$$

A distortion coefficient of $\mathrm{k}=0.2$ and the origin of radial 
distortion at pixel $(355,355)$, indicated in Fig. $4 \mathrm{~b}$, resolved the apparent curvature of the steel wires; however, the images demonstrated broadening, or magnification, of the checkerboard squares towards the top of the image. This is consistent with projection onto a screen, or camera, which is mounted lightly above the projector, commonly referred to as a 'keystone' artefact. The estimation of transformation functions for the correction of projection artefacts is comprehensively detailed in Goshtasby 1988 [18] .
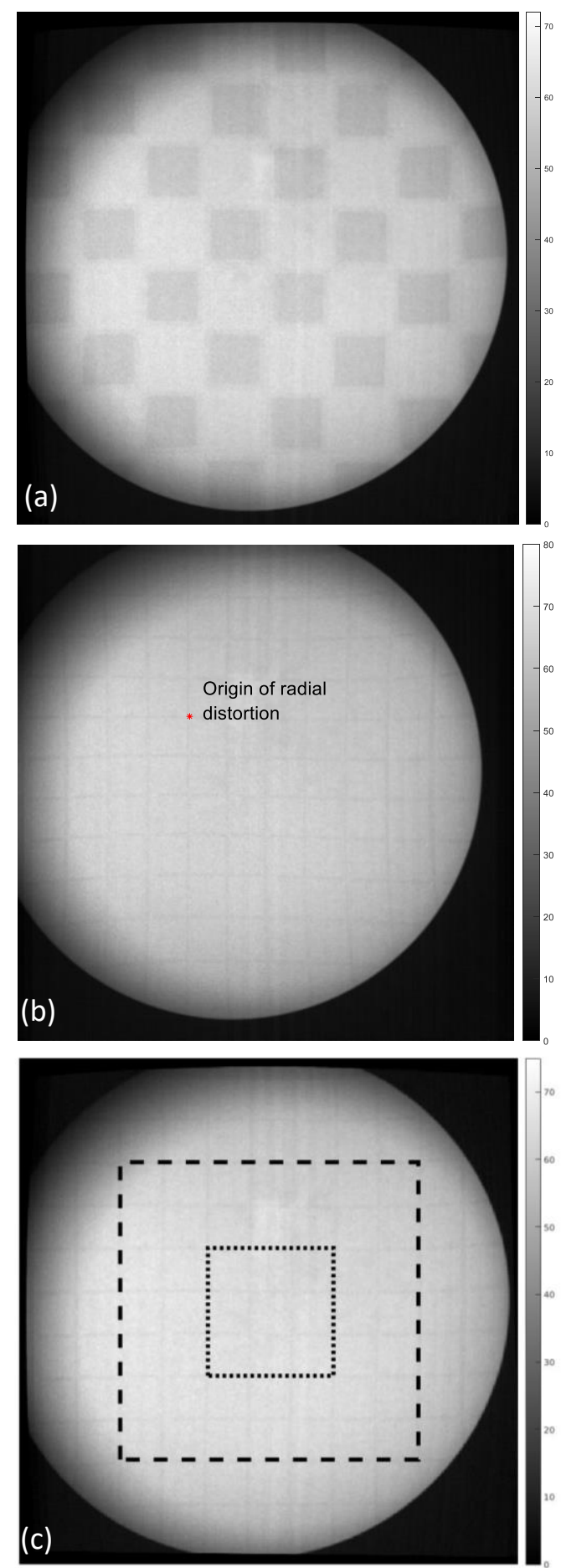

Fig. 4. Images of (a) the coarse checkerboard, (b) the fine-wire checkerboard indicating the origin of radial distortion, and (c) the fine-wire checkerboard following correction for radial and projective distortion, showing the corners of $5 \times 5$ and $7 \times 7$ square grids to demonstrate the uniformity of the corrected image.

The fine-wire checkerboard is presented following correction for radial and keystone distortion in Fig. 4c. Dashed and dotted outlines of $7 \times 7$ and $5 \times 5$ square regions respectively are overlaid to demonstrate the uniformity of the checkerboard following the two-stage transformation. The geometric transformation functions will remain valid for the experimental facility as long as the relative positioning of the X-ray source, scintillator/image intensifier and camera remain unchanged.

\section{Geometric calibration and parallax errors}

Following correction for distortion artefacts, the $7 \times 7$ square region of the checkerboard outlined in Fig. $4 \mathrm{c}(69 \times 69 \mathrm{~mm}$ in dimension) measured $692 \times 693$ pixels, corresponding to a pixel resolution of $100.7 \mu \mathrm{m}$. The observed cross-sectional areas of multiple phantom geometries of different sizes, including the steel-filled regions of phantom 2 presented in Fig. 2, are compared with their known dimensions in Fig. 5a. Measurements from 7 different calibration experiments indicated an average pixel resolution of $101.8 \mu \mathrm{m}$. The three largest objects measured (including the $7 \times 7$ checkerboard shown in Fig. 4b and the filled rings of phantom 2, see Fig. 2b) lie within $6.5 \%$ uncertainty of the calibration line. This uncertainty escalates as the size of the object diminishes, increasing to $\pm 24 \%$ for $8 \mathrm{~mm}$ steel balls.

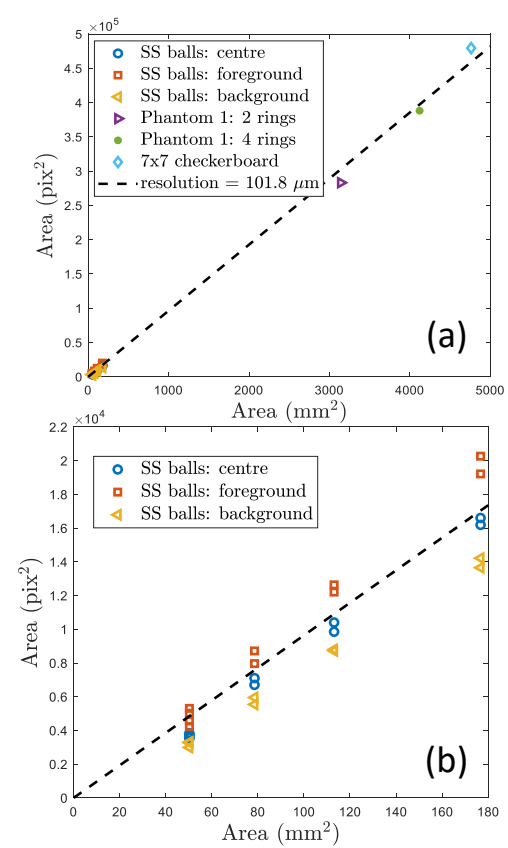

Fig. 5. (a) Calibration of pixel resolution and (b) the effects of parallax errors on the observed particle silhouette area.

The error in an object's silhouette area is liable to increase further as a result of the uncertainty in its depth location within the test section. Fig. $5 \mathrm{~b}$ demonstrates the apparent magnification of an object's observed silhouette as it is displaced within the test section towards the X-ray source. For a $15 \mathrm{~mm}$ diameter steel ball at the center of the field of view, a $40 \mathrm{~mm}$ displacement towards the foreground manifests in a 10.5 
$\%$ increase in observed diameter, while displacement $40 \mathrm{~mm}$ towards the background results in an observed $7.5 \%$ reduction in measured diameter. These measurements indicate the X-ray source to be installed around $0.46 \mathrm{~m}$ from the central axis of the test section and suggest that the observed dimension of millimetre-scale objects could be subject to a parallax uncertainty in excess of $\pm 10 \%$.

\section{ASSESSMENT OF DETECTION THRESHOLDS}

\section{A. Numerical Simulation}

The anticipated signal to noise ratio, contrast between the various phases and the limits of detection can be estimated by numerical simulation of the experimental facility in question and the associated imaging system. The MODHERATO code [9] enables the definition of a virtual representation of the Xray source, test section, test section contents, scintillator and camera configuration, generating simulated X-ray images, inclusive of statistical noise, swank noise, shot noise, dark noise, readout noise and quantization noise.

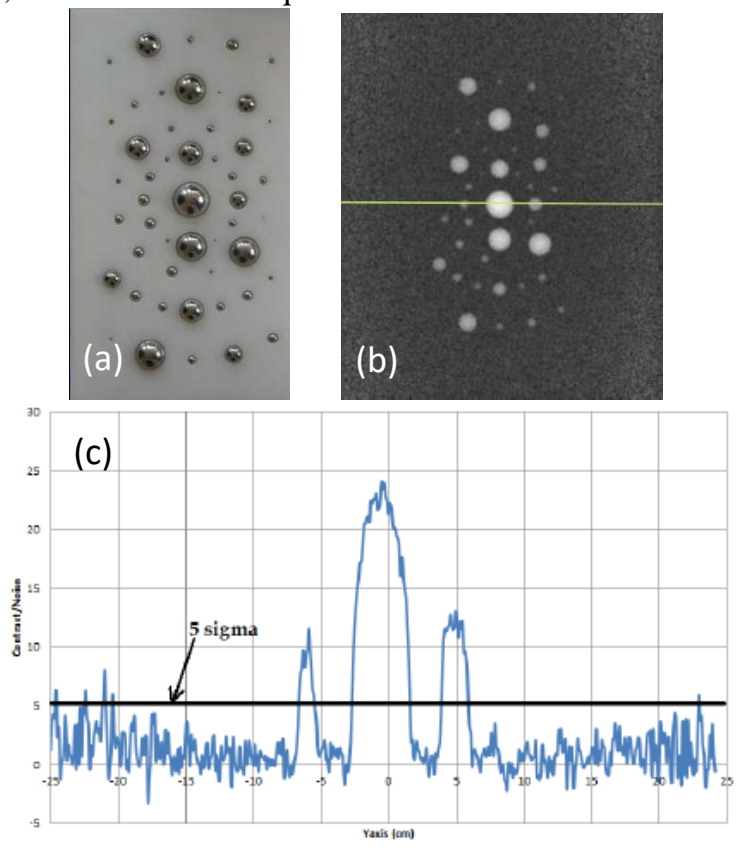

Fig. 6. Detection of metallic balls (reproduced with permission from Singh [12] ) including (a) the phantom embedded with steel balls, (b) a simulated X-ray image generated using MODHERATO and (c) the contrast to noise ratio along the analysis line in green in the top right image.

Fig. 6a presents a phantom model consisting of a plate embedded with polydisperse steel balls, which has previously been used for calibration of the KROTOS facility. This geometry, installed within the KROTOS test section, has been simulated using MODERHATO, producing the theoretical radiographic image presented in Fig. 6b. The intensity profile along the green analysis line is shown in blue in Fig. 6c. A relatively conservative threshold at 5 times the standard deviation of the imaging noise corresponded to a detection limit equivalent to $5 \mathrm{~mm}$ diameter steel balls.

\section{B. Determination of the Lower Detection Limits using Phantoms}

While numerical simulation is valuable during the design a new radioscopic system, physical phantoms are preferred for the assessment of existing experimental facilities and X-ray imaging systems. A POM insert embedded with 16 polydisperse stainless steel balls, of 1 to $15 \mathrm{~mm}$ in diameter (see Fig. 1d) was installed in phantom 1 and imaged at the MELT facility. The resulting X-ray image, presented in Fig. 7a, is shown following log-flat field normalization and following segmentation in Fig. $7 \mathrm{~b}$ and c respectively. A schematic of the steel ball locations and sizes is presented in Fig. 7d. Based on the 10 steel balls identifiable in the processed images, the lower limit of detection was found to correspond to a steel ball diameter of $4 \mathrm{~mm}$.
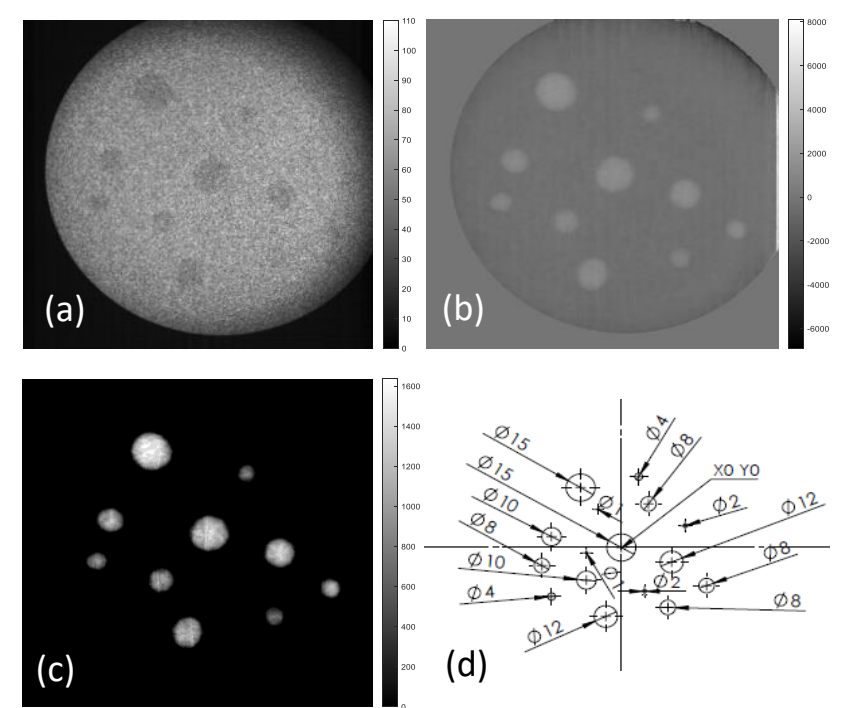

Fig. 7. The detection of a lower detectability limit using polydisperse steel ball phantoms, including (a) an unprocessed X-ray image of the phantom, (b) a denoised, log-flat field normalized and inverted image, (c) a segmented image showing the detected steel balls, and (d) a schematic of the steel ball sizes and locations.

During FCI experiments, a significant proportion of the melt fragment population is likely to be smaller than this lower limit of detection. However, given a sufficient concentration of fine particles it may be possible to detect clouds of fine particles. Fig. 8 presents a phantom employed at the KROTOS facility[8] [12] used to simulate clouds of small particles; this is the same phantom of concentric rings installed in the central annulus of phantom 2 to obtain the images acquired at the MELT facility shown in Fig. 2. The width of the concentric annuli is $20 \mathrm{~mm}$. The phantom has a steel rod at its center, representing a molten steel jet. Melt fragments are represented either by $4 \mathrm{~mm}$ diameter steel balls (Fig. 8b) with a packing fraction of $52 \%$, corresponding to a cloud density of around $3780 \mathrm{~kg} \mathrm{~m}^{-3}$, or hafnia powder (Fig. 8c), with $95 \%$ of grains below $510 \mathrm{~nm}$ and a packing fraction of $23 \%$, presenting with a cloud density of $2200 \mathrm{~kg} \mathrm{~m}^{-3}$. 
Several configurations of the phantom with 1,3 or 4 of the concentric annuli filled with steel balls or hafnia powder are presented in Fig. 8e-g and Fig. 8h-j respectively. Despite the reduced contrast with the thermoplastic coolant analogue in the case of the less dense hafnia powder, the fragment clouds occupying a single $20 \mathrm{~mm}$ wide annulus were clearly detectable, even within a vapor cloud of three-times the thickness of the particle cloud. Furthermore, the boundaries between particle clouds with $40 \mathrm{~mm}$ difference in thickness were clearly distinguishable at the KROTOS imaging facility.

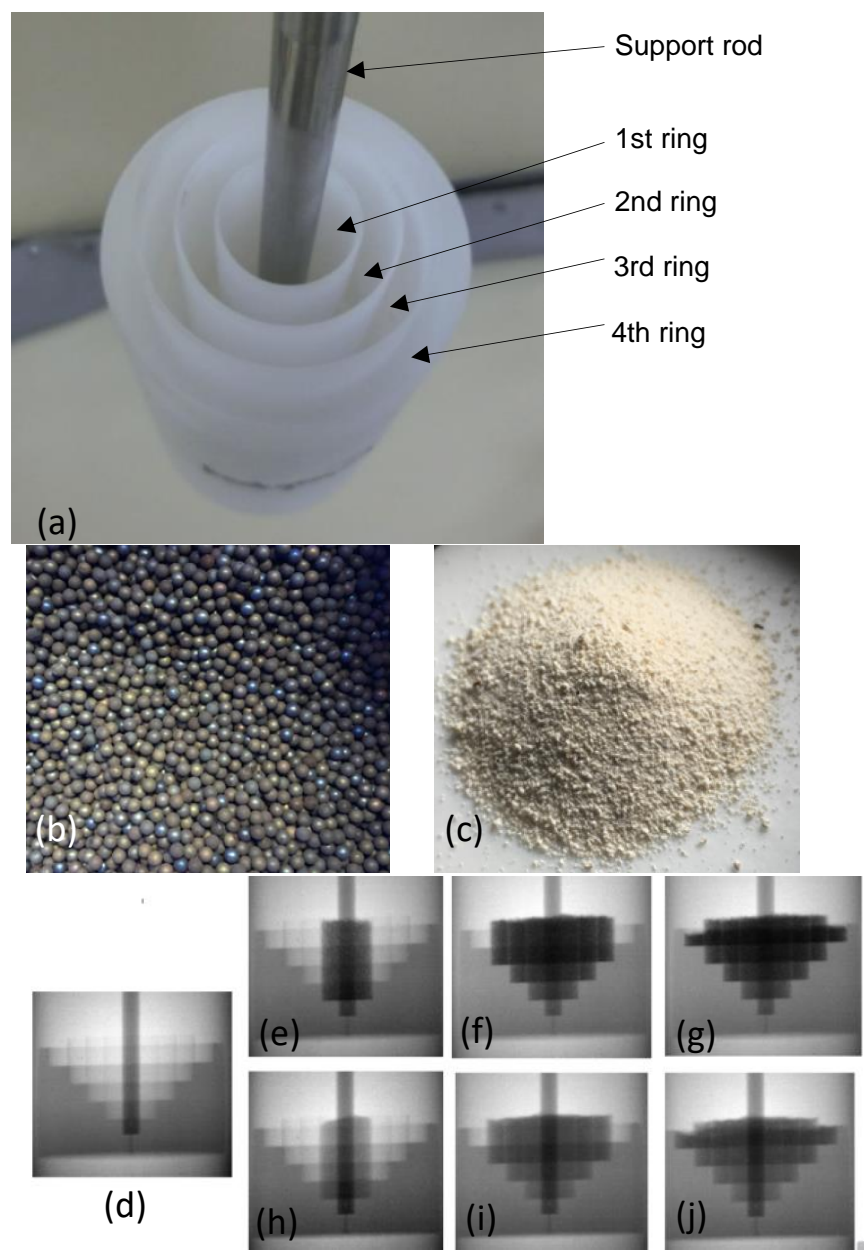

Fig. 8. Investigation of clouds of particles using an annular phantom (reproduced from [12] with permission) including (a) the phantom model, (b) 4 mm diameter steel balls, (c) fine $\mathrm{HfO}_{2}$ powder, and X-ray images of (d) the empty phantom, the phantom with (e-g) 1, 3 and 4 rings filled with steel balls, and (h-j) 1, 3 and 4 rings filled with $\mathrm{HfO}_{2}$ powder.

An additional phantom has been designed to assess the detectability of gas films around the melt jet, for investigation using the X-ray imaging system employed at the MELT facility. The phantom mimics a gaseous film around a steel jet. The tapered, or conical, steel rod at the center of the phantom is surrounded by a void film which increases from a thickness of $0 \mathrm{~mm}$ at the base, to $6.75 \mathrm{~mm}$ at the top. The phantom design and a corresponding X-ray image of the phantom are presented in Fig. 9. It was determined that vapor films of as little as 1.7 $\mathrm{mm}$ thickness were detectable, however the contrast to noise ratio fell significantly for film thicknesses below $3.75 \mathrm{~mm}$.
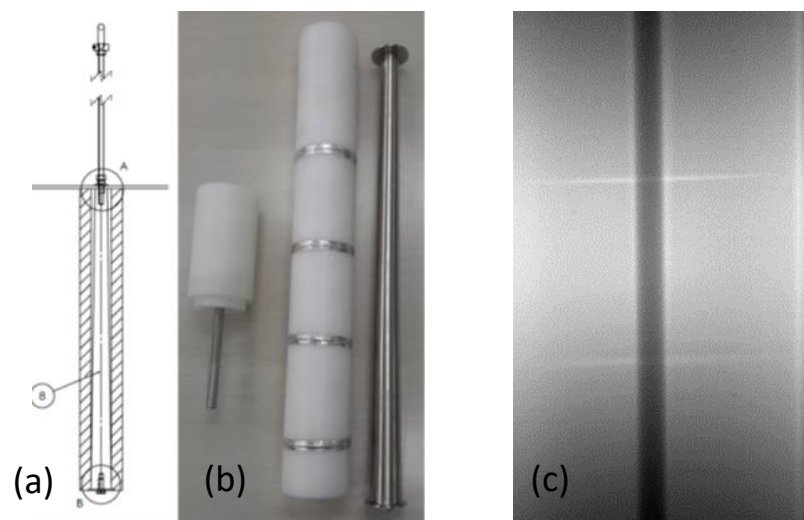

Fig. 9. (a) A schematic, and (b) the components of a phantom used to determine the detection limit for vapor films, with (c) the corresponding X-ray image acquired at the MELT facility.

\section{CONCLUSIONS}

High-speed fan beam X-ray imaging systems employed for the visualization of transient thermal hydraulic experiments such as fuel-coolant interactions are subject to significant imaging artefacts, such as noise, loss of intensity towards the border of the image, and lens distortion. Image processing is necessary to suppress these artefacts and achieve homogeneous illumination of the imaging window to detect melt fragments and to achieve a homogenous pixel resolution for quantitative assessment of the images. Image calibration is highly recommended to determine the extent of these imaging artefacts, the uncertainties associated with quantitative measurements and the lower limit of detectability for small particles and vapor films. A series of versatile phantom model designs have been presented to assess the various capabilities of such X-ray imaging systems, using steel balls and hafnia powder as analogues for different melt phases, thermoplastic materials such as polyoxymethylene or polyethylene to represent liquid sodium coolant, and voids for the coolant vapor.

Calibration experiments using phantoms enabled the determination of transform functions to correct for the lens distortion at the MELT facility and achieve images with a homogeneous resolution of $102 \mu \mathrm{m}$. The lower limit of detectability was found to correspond to individual melt fragments in the order of $4 \mathrm{~mm}$ diameter and vapor films of around 1.7-3.75 mm thickness. Characterization of the size of the melt fragment population was subject to uncertainties which increased with diminishing particle size, rising to $\pm 24 \%$ for 8-mm diameter steel spheres. Assuming the jet to disperse to a radius of $40 \mathrm{~mm}$ during pre-mixing, measurements may be subject to a further parallax uncertainty in the order of $\pm 10 \%$ due to the apparent magnification of foreground objects.

\section{ACKNOWLEDGMENT}

This work was carried out within the arrangement between CEA, FRAMATOME, JAEA, MHI and MFBR entered into force January 1, 2020.

The calibration experiments at the MELT facility were supported by the Japanese Ministry of Economy, Trade and Industry through the "Technical Development Program on Fast 
Reactor International Cooperation" and the "Technical Development Program on a Common Base for Fast Reactors".

The authors wish to thank the JAEA's MELT team for performing the $\mathrm{X}$-ray image acquisition and Frédéric Compagnon for his work on the design of the phantom models used in this study.

\section{REFERENCES}

[1] M.L. Corradini, B.J. Kim and M.D. Oh, "Vapor explosions in light water reactors: A review of theory and modelling", Prog. Nucl. Ener. vol. 22, no. 1, pp. 1-117, 1988, 10.1016/0149-1970(88)90004-2.

[2] R. Meignien, B. Raverdy, M. Buck, G. Pohlner, P. Kudinov, W. Ma, C. Brayer, P. Piluso, S.-W. Hong, M. Leskovar, M. Uršič, G. Albrecht, I. Lindholm and I. Ivanov, "Status of steam explosion understanding and modelling", Ann. Nucl. Ener., vol. 74, pp. 125-133, 2014, 10.1016/j.anucene.2014.07.008.

[3] P. Shen, W. Zhou, N. Cassiault-Louis, C. Journeau, P. Piluso and Y. Liao, "Corium behavior and steam explosion risks: a review of experiments", Ann. Nucl. Ener. vol. 121, pp. 162-176, Nov. 2018, 10.1016/j.anucene.2018.07.029.

[4] G.Berthoud, H. Jacobs and B. Knowles, "Analysis of large scale $\mathrm{UO}_{2} \mathrm{Na}$ interactions performed in Europe", IAEA-IWGFR technical committee meeting on material-coolant interactions and material movement and relocation in liquid metal fast reactors, O-arai, Ibaraki (Japan), June 1994, pp. 127-165.

[5] K. Matsuba, K. Kamiyama, J. Toyooka, Y. Tobita, V.A. Zuyev, A. A. Kolodeshnikov and Y.S. Vassiliev, "Experimental discussion on fragmentation mechanisms of molten oxide discharged into a sodium pool”, Mech. Eng. J. vol. 3, no. 3, pp. 15-00595, 2016, 10.1299/mej.15-00595.

[6] M. Johnson, C. Journeau, K. Matsuba, Y. Emura and K. Kamiyama, Characterization of high-temperature nuclear fuel-coolant interactions through X-ray visualization and image processing”, Ann. Nucl. Ener., vol. 151, pp. 107881, Feb. 2021, 10.1016/j.anucene.2020.107881.

[7] H.S. Park, R.C. Hansson, B.R. Sehgal, Fine fragmentation of molten droplet in highly subcooled water due to vapor explosion observed by $\mathrm{X}$ ray radioscopy, Exp. Therm. Fluid Sci., vol. 29, pp. 351-361, 2005.
[8] C. Brayer, D. Grishchenko, N. Cassiaut-Louis, P. Piluso, "Application of $\mathrm{X}$-Ray radioscopy for investigations of the 3-phase-mixture resulting from the fragmentation of a high temperature molten material jet in water", in: $8^{\text {th }}$ Int. Conf. Multiphase Flow, ICMF-2013, Jeju, Korea, 2013,10.1016/j.expthermflusci.2004.05.013.

[9] L. Berge, N. Estre, D. Tisseur, E. Payan, D. Eck, V. Bouyer, N. Cassiaut-Louis, C. Journeau, R. Le Tellier, S. Singh, E. Pluyette, "Fast Energy X-Ray Imaging for Severe Accidents on the Future PLINIUS-2 Platform", IEEE Trans. Nucl. Sci. vol. 65, pp. 2573-2581, 2018.

[10] M. Zabiego, C. Fochesato, "Corium-sodium interaction the development of the SCONE software", in: NURETH-17 - 17th International Topical Meeting on Nuclear Reactor Thermal Hydraulics, Xi-An, China, 2017,

[11] A.R. Cowen, A.G. Davies, M.U. Sivananthan, "The design and imaging characteristics of dynamic, solid-state, flat-panel X-ray image detectors for digital fluoroscopy and fluorography", Clinical Radiology, voL 63, pp. 1073-1085,

[12] S. Singh, "X-Ray diagnostics on corium-sodium interactions during a severe accident scenario", PhD dissertation, University Paris-Saclay, 2019, https://hal.archives-ouvertes.fr/tel-02897391

[13] A. Danielyan, V. Katkovnik, K. Egiazarian, K.. BM3D frames and variational image deblurring. IEEE Trans. Image Proc. vol. 21(4), pp. 1715-1728, 2012, https://doi.org/10.1109/TIP.2011.2176954

[14] A. Buades, B. Coll, J. M. Morel, "A Review of Image Denoising Algorithms, with a New One", Multiscale Model. Simul., vol. 4, no. 2, pp. 490-530, 2005.

[15] I. Frosio J. Kautz, "Statistical Nearest Neighbors for Image Denoising," IEEE Trans. Image Process., vol. 28, no. 2, pp. 723-738, 2019.

[16] V. Van Nieuwenhove, J. De Beenhouwer, F. De Carlo, L. Mancini, F. Marone, J. Sijbers, "Dynamic intensity normalization using eigen flat fields in X-ray imaging," Opt. Express, vol. 23, no. 21, Art no. 27975, 2015.

[17] G. Vass and T. Perlaki, "Applying and removing lens distortion in post production," in 2nd Hungarian Conference on Computer Graphics and Geometry, 2003, pp. 9-16.

[18] A. Goshtasby, "Image registration by local approximation methods," Image Vis. Comput., vol. 6, pp. 255-261, 1988. 